\title{
VARIABILIDADE ESPACIAL DE ATRIBUTOS QUÍMICOS DO SOLO SOB DIFERENTES USOS E MANEJOS ${ }^{(1)}$
}

\author{
Eloiza Gomes Silva Cavalcante ${ }^{(2)}$, Marlene Cristina Alves ${ }^{(3)}$, \\ Zigomar Menezes de Souza ${ }^{(4)}$ \& Gener Tadeu Pereira ${ }^{(5)}$
}

\begin{abstract}
RESUMO
O uso e manejo do solo e da cultura são importantes condicionadores da variabilidade de atributos do solo. O trabalho foi desenvolvido em Selvíria (MS), com o objetivo de avaliar a variabilidade espacial do pH, potássio (K), cálcio (Ca), magnésio (Mg) e saturação por bases (V) em Latossolo Vermelho sob diferentes usos e manejos. Os solos foram amostrados em malha, com intervalos regulares de $2 \mathrm{~m}$, perfazendo o total de 64 pontos, nas profundidades de 0,0-0,1 e 0,1-0,2 m, nas seguintes áreas: vegetação natural (Cerrado), plantio direto, plantio convencional e pastagem. As maiores variabilidades, medidas por meio do coeficiente de variação, foram observadas para $\mathrm{K}, \mathrm{Mg}$ e Ca; $\mathrm{pH}$ apresentou o menor coeficiente de variação nos diferentes usos e manejo do solo, e o atributo $V$, coeficiente de variação médio. Os sistemas preparo convencional e pastagem apresentaram os menores alcances quando comparado aos sistemas Cerrado e plantio direto.
\end{abstract}

Termos de indexação: preparo do solo, amostragem, geoestatística, agroecossistemas, Latossolo.

\section{SUMMARY: SPATIAL VARIABILITY OF CHEMICAL ATTRIBUTES OF A SOIL UNDER DIFFERENT USES AND MANAGEMENTS}

The use and management of soil and crop condition the variability of soil attributes directly. This study was conducted in Selviria-MS, Brazil with the objective of evaluating

\footnotetext{
(1) Parte da Tese de Mestrado do primeiro autor, financiada pela FAPESP. Recebido para publicação em maio de 2006 e aprovado em julho de 2007.

${ }^{(2)}$ Mestranda do Programa de Pós-Graduação da Faculdade de Engenharia, Universidade Estadual Paulista - UNESP. Caixa Postal 31, CEP 15385-000 Ilha Solteira (SP). E-mail: cavalcante@agr.feis.unesp.br

(3) Professora Livre Docente da Faculdade de Engenharia, Campus de Ilha Solteira, UNESP. E-mail: mcalves@agr.feis.unesp.br

(4) Professor da Faculdade de Engenharia Agrícola, Campus de Ilha Solteira, Universidade de Campinas - FEAGRI/UNICAMP. Cidade Universitária Zeferino Vaz, Caixa Postal 6011, CEP 13083-875 Campinas (SP). E-mail: zigomarms@agr.unicamp.br

(5) Professor da Faculdade de Ciências Agrárias e Veterinárias, Universidade Estadual Paulista - FCAV/UNESP. Rod. Paulo Donato Castellane s/n, CEP 14884-900 Jaboticabal (SP). E-mail: genertp@fcav.unesp.br
} 


\begin{abstract}
the spatial variability of $\mathrm{pH}$, potassium $(\mathrm{K})$, calcium $(\mathrm{Ca})$, magnesium $(\mathrm{Mg})$ and base saturation (\% BS) in an Oxisol under different use and management conditions. Soil samples were collected in a grid, in regular $2 \mathrm{~m}$ intervals, at 64 grid points, at depths of $0.0-0.1 \mathrm{~m}$ and $0.1-0.2 \mathrm{~m}$, from areas of: native cerrado vegetation (savannah), annual crops under no-tillage, annual crops under conventional tillage, and pasture. The highest variabilities, as determined by the coefficient of variation, were observed for $\mathrm{K}, \mathrm{Mg}$ and $\mathrm{Ca}$, while the lowest coefficient of variation was found for $\mathrm{pH}$ in the different uses and management conditions. A medium coefficient of variation was observed for the $\% B S$. The ranges of soils under conventional tillage and pasture systems were smaller than those under native vegetation and no-tillage.
\end{abstract}

Index terms: tillage, sampling, geostatistics, agroecosystems, Oxisol.

\section{INTRODUÇÃO}

O conhecimento da variabilidade dos atributos do solo sob diferentes usos e manejos constitui-se numa importante meta para que se possa empregar manejo mais adequado, considerando a aplicação de fertilizantes, estratégias de amostragem e planejamento de delineamento de pesquisa em campo (Bhatti et al., 1991). A variabilidade espacial dos solos surge desde a sua formação e continua após eles atingirem estado de equilíbrio dinâmico. Por ser o solo um sistema extremamente heterogêneo, o conhecimento de sua variabilidade espacial é imprescindível, devendo ser considerado no momento da amostragem. Devido às várias combinações a que estão sujeitos os elementos do solo e às constantes reações químicas que ocorrem na solução do solo, os atributos químicos apresentam maior variação do que os físicos e, conseqüentemente, necessitam de maior número de amostras para estimá-los dentro da mesma área (Beckett \& Webster, 1971; Jacob \& Klutte, 1976).

O uso do solo, com o passar do tempo, conduz a aumento na sua heterogeneidade, por meio dos processos de desmatamento, preparo do solo, rotações de cultura, localização de aplicação de fertilizantes; como estes são aplicados em faixas ou em linhas, isso faz com que o sistema de amostragem varie consideravelmente. Segundo Salviano et al. (1998), os sistemas de manejo conservacionistas criam ambiente no solo diferente daquele encontrado no sistema convencional, resultante dos efeitos dos resíduos superficiais e da reduzida movimentação do solo sobre os atributos físicos, químicos e biológicos. Portanto, em estudos agronômicos do sistema soloágua-planta deve-se considerar a variabilidade dos solos, pois os fatores e processos de sua formação que atuaram ao longo do tempo imprimiram-lhe variabilidades naturais; estas, somadas ao manejo realizado pelo homem, acentuam a variabilidade dos atributos do solo (Albuquerque et al., 1996).

A geoestatística vem apresentando aplicação crescente na ciência do solo, tornando-se ferramenta adicional no estudo de seus atributos espacialmente correlacionados, exatamente porque incorpora em si a possibilidade de se estudar o comportamento da variabilidade espacial, permitindo a interpretação dos resultados com base na estrutura dessa variabilidade, além de poder também quantificar o seu tamanho. Como no Brasil a aplicação de insumos é baseada em teores médios da fertilidade do solo, os quais podem ser subestimados ou superestimados, o conhecimento detalhado da variabilidade espacial dos atributos da fertilidade, por meio da geoestatística, pode otimizar a aplicação localizada de corretivos e fertilizantes, melhorando dessa maneira o controle do sistema de produção das culturas e contaminações ambientais.

O manejo do solo e o da cultura são importantes condicionadores da variabilidade de atributos do solo. Solos de mesma classe taxonômica, considerados relativamente homogêneos, podem apresentar variação em seus atributos como resultado da aplicação de diferentes práticas de manejo. Da mesma maneira, solos de classes diferentes, submetidos ao mesmo manejo, podem apresentar atributos semelhantes (Bouma et al., 1999). Portanto, o objetivo do presente trabalho foi estudar a variabilidade espacial de atributos químicos em Latossolo Vermelho, em diferentes condições de uso e manejo.

\section{MATERIAL E MÉTODOS}

O estudo foi realizado na Fazenda de Ensino e Pesquisa da Faculdade de Engenharia, campus de Ilha Solteira, da Universidade Estadual Paulista "Júlio de Mesquita Filho", situada à margem direita do rio Paraná, no município de Selvíria (MS), localizado nas coordenadas geográficas $20^{\circ} 22^{\prime} \mathrm{S}$ e $51^{\circ} 22^{\prime} \mathrm{W}$, numa altitude média de $335 \mathrm{~m}$. O tipo climático, segundo Köppen, é Aw, caracterizado como tropical úmido com estação chuvosa no verão e seca no inverno, estando a umidade relativa dos meses mais chuvosos entre 60 e $80 \%$. O solo foi classificado como Latossolo Vermelho hipodistrófico argiloso A moderado álico, caulinítico férrico compactado, muito profundo, moderadamente ácido (LVd) (Embrapa, 1999).

A área com vegetação natural, onde predominam as formas arbustivas em solo profundo e pouco fértil, conhecida como Cerrado, foi desmatada em 1978, com 
plantio da pastagem neste ano, instalação do preparo convencional em 1980 e implantação do sistema plantio direto em 1990. O plantio direto apresenta seqüência de culturas com leguminosas e gramíneas, como feijão (Phaseolus vulgaris), milho (Zea mays L.), soja (Glycine Max (L) Merril) e aveia-preta (Avena strigosa Schieb), e o manejo de plantas invasoras tem sido efetuado com herbicidas, sendo cultivada com este sistema há 16 anos. O preparo convencional, realizado há 26 anos, consta de gradagem pesada (aradora) e gradagem leve (niveladora), com cultivo de culturas anuais. Efetuou-se a calagem antes da instalação dos sistemas plantio direto e preparo convencional, com o objetivo de elevar a saturação por bases a $60 \%$. As culturas de feijão, milho e soja receberam adubação anual de $200 \mathrm{~kg} \mathrm{ha}^{-1}$ da fórmula 4-30-10, enquanto a área de pastagem (Brachiaria decumbens) só recebeu calagem e adubação na implantação e reformas - área com 28 anos de cultivo.

A amostragem do solo foi realizada nos pontos de cruzamento de malha, com intervalos regulares de $2 \mathrm{~m}$, perfazendo o total de 64 pontos $(14 \times 14 \mathrm{~m})$, sendo coletada uma amostra por ponto (Figura 1), nas profundidades de $0,0-0,1$ e $0,1-0,2 \mathrm{~m}$. $\mathrm{Na}$ caracterização química do solo foi determinada a acidez ativa ( $\mathrm{pH}$ em $\mathrm{CaCl}_{2}$ ), potenciometricamente. Os valores dos atributos $\mathrm{Ca}$ e $\mathrm{Mg}$ disponível foram extraídos utilizando o método da resina trocadora de íons, proposto por Raij et al. (2001). Com base nos resultados das análises químicas, foi calculado o valor da pecentagem de saturação por bases do solo (V, \%).

A variabilidade do solo foi avaliada pela análise exploratória dos dados, calculando-se a média, mediana, desvio-padrão, coeficiente de variação, coeficiente de assimetria e coeficiente de curtose. A

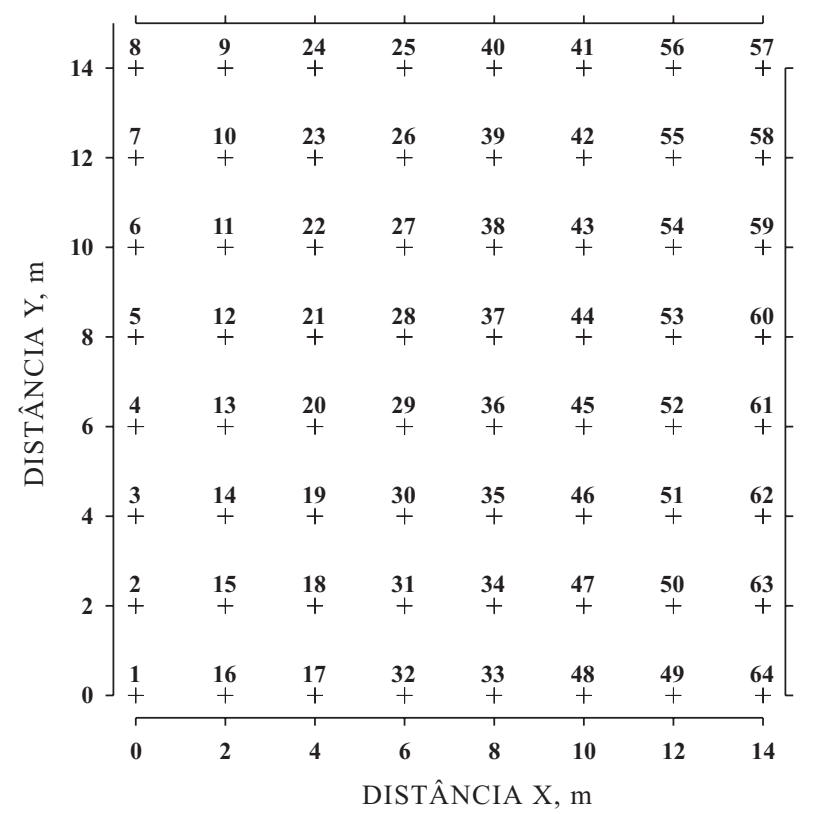

Figura 1. Esquema de amostragem nos diferentes sistemas de uso e manejo do solo. hipótese de normalidade dos dados foi verificada pelo teste de Kolmogorov-Smirnov. A análise descritiva e o teste de normalidade dos dados foram realizados utilizando-se o programa SAS (Schlotzhaver \& Littell, 1997). A dependência espacial foi avaliada através da geoestatística, conforme Vieira et al. (1983), por meio do cálculo da semivariância. A análise geoestatística é baseada na suposição de que medições separadas por distâncias pequenas são mais semelhantes umas às outras do que aquelas separadas por distâncias maiores. A semivariância é, por definição, dada por:

$$
\gamma(\mathrm{h})=\frac{1}{2} \mathrm{E}\left[\mathrm{Z}\left(\mathrm{x}_{\mathrm{i}}\right)-\mathrm{Z}\left(\mathrm{x}_{\mathrm{i}}+\mathrm{h}\right)\right]^{2}
$$

a qual é uma função do vetor h e, portanto, depende da magnitude e da direção $h$.

A semivariância é estimada pela expressão a seguir:

$$
\gamma^{*}(h)=\frac{1}{2 N(h)} \sum_{i=1}^{N(h)}\left[Z\left(x_{i}\right)-Z\left(x_{i}+h\right)\right]^{2}
$$

sendo $\mathrm{N}(\mathrm{h})$ : número de pares de valores medidos, $\mathrm{z}(\mathrm{xi})$ e $z(x i+h)$, separados por um vetor distância $h$; e z(xi) e $z(x i+h)$ : valores da i-ésima observação da variável regionalizada, coletados nos pontos xi e $x i+h$ $(i=1, \ldots, n)$, separados pelo vetor $h$. O gráfico de $\gamma^{*}(h)$ em função dos valores correspondentes de $\mathrm{h}$ é denominado semivariograma. Do ajuste do modelo matemático aos valores calculados de $\gamma^{*}(\mathrm{~h})$, são estimados os coeficientes do modelo teórico para o semivariograma (efeito pepita, $\mathrm{C}_{0}$; patamar, $\mathrm{C}_{0}+\mathrm{C}_{1}$; alcance, a). A razão entre o efeito pepita e o patamar permite a classificação e a comparação entre os atributos do solo (Trangmar et al., 1985). Esta razão foi utilizada para definir as classes distintas de dependência espacial das variáveis do solo, utilizandose a classificação de Cambardella et al. (1994). Considera-se dependência espacial forte quando a razão é menor ou igual a $25 \%$; dependência espacial moderada quando a razão é superior a $25 \%$ e menor ou igual a $75 \%$; e dependência fraca quando a razão é maior que $75 \%$.

Foram ajustados aos dados os seguintes modelos: (a) esférico (Esf), $\gamma^{*}(\mathrm{~h})=\mathrm{C}_{0}+\mathrm{C}_{1}\left[1,5(\mathrm{~h} / \mathrm{a})-0,5(\mathrm{~h} / \mathrm{a})^{3}\right]$ para $0<\mathrm{h}<$ a e $\gamma^{*}(\mathrm{~h})=\mathrm{C}_{0}+\mathrm{C}_{1}$ para $\mathrm{h}>\mathrm{a}$; (b) exponencial $(\operatorname{Exp}), \gamma^{*}(\mathrm{~h})=\mathrm{C}_{0}+\mathrm{C}_{1}[1-\exp (-3 \mathrm{~h} / \mathrm{a})]$ para $0<\mathrm{h}<\mathrm{d}$, em que d é a distância máxima na qual o semivariograma é definido; e (c) gaussiano (Gau), $\gamma^{*}(\mathrm{~h})=\mathrm{C}_{0}+\mathrm{C}_{1}\left[1-\exp \left(-3 \mathrm{~h}^{2} / \mathrm{a}^{2}\right)\right]$. Esses modelos foram ajustados utilizando-se o programa $\mathrm{GS}^{+}$ (Robertson, 1998).

\section{RESULTADOS E DISCUSSÃO}

De acordo com os limites estabelecidos para os níveis de fertilidade do solo no Estado de São Paulo (Raij et al., 1997), os valores médios dos atributos químicos nas profundidades de $0,0-0,1$ e $0,1-0,2 \mathrm{~m}$ 
apresentaram-se baixos para $\mathrm{pH}$ em todos os sistemas de usos e manejos estudados; os valores de $\mathrm{K}, \mathrm{Ca}, \mathrm{Mg}$ e V foram baixos para o Cerrado e pastagem e oscilaram de médio a alto para os sistemas plantio direto e preparo convencional (Quadro 1). Resultados semelhantes foram verificados por Souza \& Alves (2003), estudando os atributos químicos de um
Latossolo Vermelho distrófico de Cerrado sob diferentes usos e manejos. Os sistemas plantio direto e convencional apresentaram os maiores valores de $\mathrm{K}$, $\mathrm{Ca}, \mathrm{Mg}$ e V quando comparados aos sistemas Cerrado e pastagem, uma vez que os dois primeiros envolviam o uso de culturas anuais, nas quais foram feitas adubações com fertilizantes minerais.

Quadro 1. Estatística descritiva para pH, potássio, cálcio, magnésio e saturação por bases de amostras coletadas na malha nas profundidades de 0,0-0,1 e 0,1-0,2 m, em diferentes uso e manejo do solo

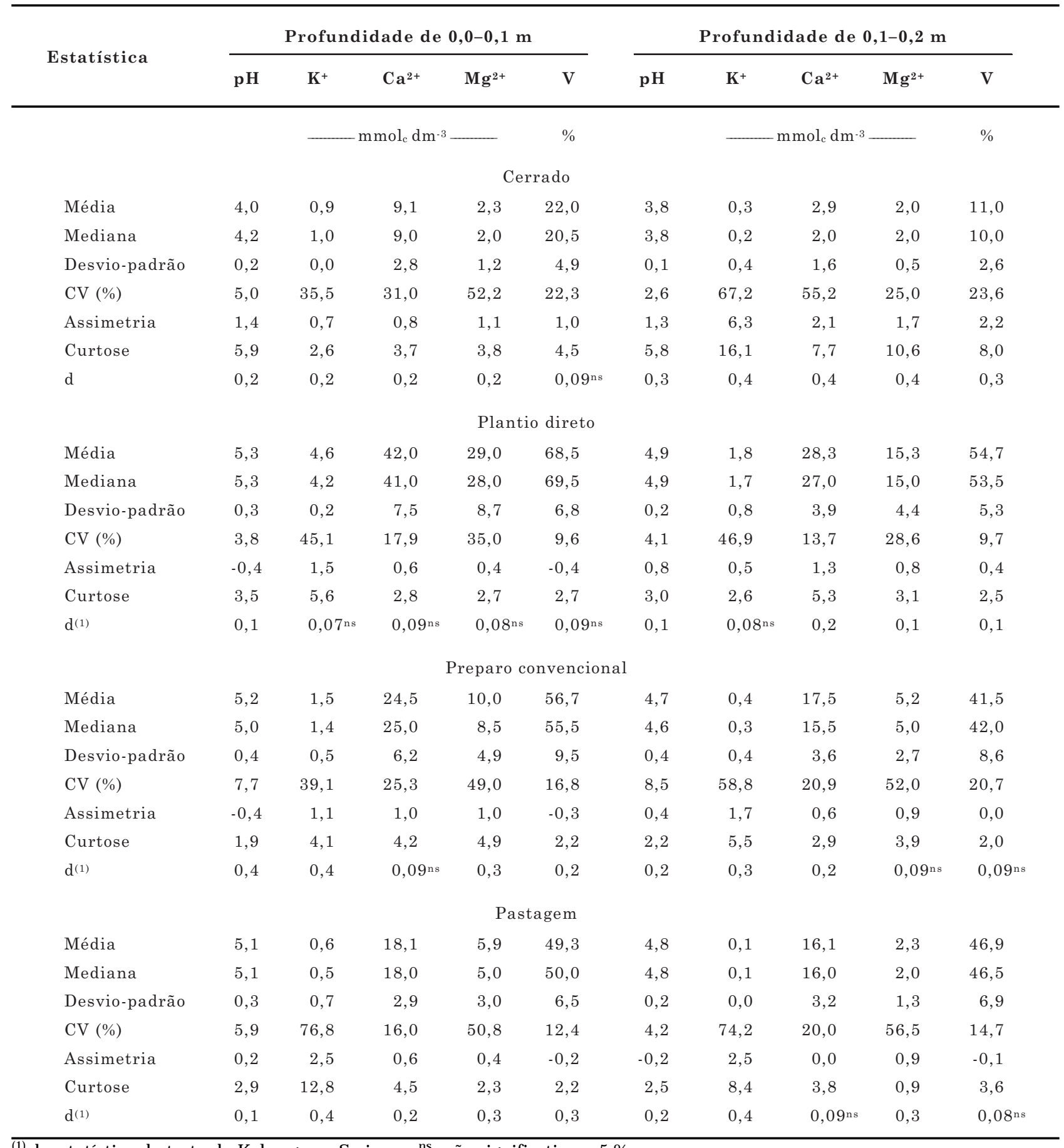

${ }^{(1)} \mathrm{d}$ : estatística do teste de Kolmogorov-Smirnov, ${ }^{\text {ns: }}$ não-significativo a $5 \%$. 
Observou-se diminuição dos valores dos atributos químicos em profundidade para todos os sistemas estudados, concordando com os resultados obtidos por Bayer \& Mielniczuk (1997) e Souza \& Alves (2003). Esse comportamento é explicado, no sistema plantio direto, pelo não-revolvimento, favorecendo o acúmulo de nutrientes na superfície; na área com pastagem, os baixos valores para os atributos químicos em estudo devem-se ao estádio avançado de degradação, fato comum na região.

Os resultados referentes à análise descritiva para os valores de $\mathrm{pH}$ e teores de $\mathrm{K}, \mathrm{Ca}, \mathrm{Mg}$ e V são apresentados no quadro 1 . Os valores de assimetria $\mathrm{e}$ curtose demonstram distribuições assimétricas para os atributos químicos em estudo. Segundo Isaaks \& Srivastava (1989), o coeficiente de assimetria é mais sensível a valores extremos do que a média e o desviopadrão, uma vez que um único valor pode influenciar fortemente esse coeficiente, pois os desvios entre cada valor e a média são elevados à terceira potência.

Apesar da ocorrência de algumas distribuições assimétricas, os valores da média e mediana de todos os atributos químicos estudados são próximos, mostrando que os dados não apresentam assimetria acentuada; conforme Little \& Hills (1978), quando os valores da média, mediana e moda são semelhantes, os dados apresentam ou se aproximam da distribuição normal. Isso pode ser um indicativo de que as medidas de tendência central não são dominadas por valores atípicos na distribuição (Cambardella et al., 1994), demonstrando que todos os atributos envolvidos no estudo estão se aproximando de uma distribuição normal - sinal de que os dados estão adequados para uso da geoestatística.

Os resultados referentes ao teste KolmogorovSmirnov indicaram normalidade no Cerrado para o $\mathrm{V} \%$ na profundidade de $0,0-0,1 \mathrm{~m}$; para as variáveis $\mathrm{K}, \mathrm{Ca}, \mathrm{Mg}$ e V na profundidade de 0,0-0,1 m; e K na profundidade de $0,1-0,2 \mathrm{~m}$, no sistema plantio direto; no preparo convencional, $\mathrm{o} \mathrm{Ca}$ apresentou distribuição normal na profundidade de $0,0-0,1 \mathrm{~m}$; o $\mathrm{Mg}$ e V, na profundidade de $0,1-0,2 \mathrm{~m}$; e as variáveis Ca e V, na profundidade de $0,1-0,2 \mathrm{~m}$, na área com pastagem. Carvalho et al. (2003), estudando a variabilidade dos atributos químicos do solo, encontraram distribuição normal para $\mathrm{V} \%$, e o $\mathrm{pH}, \mathrm{Ca}$ e $\mathrm{Mg}$ mostraram distribuição lognormal. Carvalho et al. (2002) utilizaram a geoestatística na determinação da variabilidade espacial de atributos químicos e verificaram que $40 \%$ das 30 combinações possíveis (cinco variáveis, duas profundidades e três sistemas de preparo) apresentaram valores de assimetria e curtose não compatíveis com a distribuição normal.

A normalidade dos dados não é exigência da geoestatística. Mais importante que a normalidade dos dados é a ocorrência ou não do chamado efeito proporcional, em que a média e a variabilidade dos dados são constantes na área de estudo. Esse fato não ocorreu no presente estudo, pois os semivariogramas apresentaram patamares bem definidos e a distribuição não apresentou caudas muito alongadas, o que poderia comprometer as estimativas da krigagem, as quais são baseadas nos valores médios (Isaaks \& Srivastava, 1989).

De acordo com a classificação do coeficiente de variação (CV), proposta por Warrick \& Nielsen (1980), o $\mathrm{pH}$ nas profundidades de $0,0-0,1$ e $0,1-0,2 \mathrm{~m}$ apresentou baixo CV (<12\%) em todos os sistemas de usos estudados (Quadro 1). O Ca apresentou CV médio (12 a $24 \%$ ) no sistema plantio direto e área com pastagem, nas profundidades estudadas, e, no preparo convencional, na profundidade de $0,1-0,2 \mathrm{~m}$. No Cerrado, em ambas as profundidades, e no preparo convencional na profundidade de $0,0-0,1 \mathrm{~m}$, o Ca apresentou CV alto (>24\%). O K e Mg mostraram CV alto nas profundidades estudadas em todos os sistemas de uso e manejo do solo. Já o V \% apresentou CV médio no Cerrado, preparo convencional e área com pastagem e CV baixo no sistema plantio direto nas profundidades avaliadas.

Segundo Vanni (1998), coeficiente de variação maior que $35 \%$ revela que a série é heterogênea e a média tem pouco significado. Se for maior que $65 \%$, a série é muito heterogênea e a média não tem significado algum. Contudo, se for menor que $35 \%$, a série é homogênea e a média tem significado, podendo ser utilizada como representativa da série de onde foi obtida. Dessa forma, pode-se dizer que o K, nas profundidades de $0,0-0,1$ e $01-0,2 \mathrm{~m}$ em todos os sistemas de uso e manejo estudados, o $\mathrm{Mg}$, na profundidade de $0,0-0,1 \mathrm{~m}$ em todos os sistemas e na profundidade de $0,1-0,2 \mathrm{~m}$ para o preparo convencional e área com pastagem, e o $\mathrm{Ca}$, na profundidade de $0,1-0,2 \mathrm{~m}$ no Cerrado, apresentaram série de dados heterogêneos e média com pouco significado.

$\mathrm{O}$ alto valor de $\mathrm{CV}$ para os atributos químicos do solo é provavelmente devido aos efeitos residuais de adubações anteriores, visto que geralmente a adubação ocorre na linha de plantio, enquanto a correção da acidez do solo, bem como a deposição dos restos culturais, é realizada a lanço, não ocorrendo homogeneização sobre a área. Segundo Silva et al. (2003), mesmo as aplicações a lanço, seguidas de revolvimento do solo para incorporação do calcário, geram variabilidade no solo.

$\mathrm{Na}$ seleção dos modelos dos semivariogramas foram considerados os valores de $\mathrm{R}^{2} \mathrm{e} \mathrm{SQR}$ (Quadro 2). Segundo Robertson (1998), a SQR é um parâmetro mais robusto do que $o R^{2}$ e propicia uma medida exata de qual modelo se ajusta melhor aos dados, e o programa $\mathrm{GS}^{+}$utiliza a combinação de parâmetros do semivariograma para minimizar a SQR para cada modelo.

Os resultados da análise geoestatística mostraram que os atributos químicos estudados apresentaram dependência espacial nas duas profundidades, com exceção das variáveis $\mathrm{pH}$ e K na área com pastagem, 
Quadro 2. Modelos e parâmetros estimados dos semivariogramas experimentais para o pH, potássio, cálcio, magnésio e saturação por bases de amostras coletadas na malha nas profundidades de 0,0-0,1 e 0,1$0,2 \mathrm{~m}$, em diferentes uso e manejo do solo

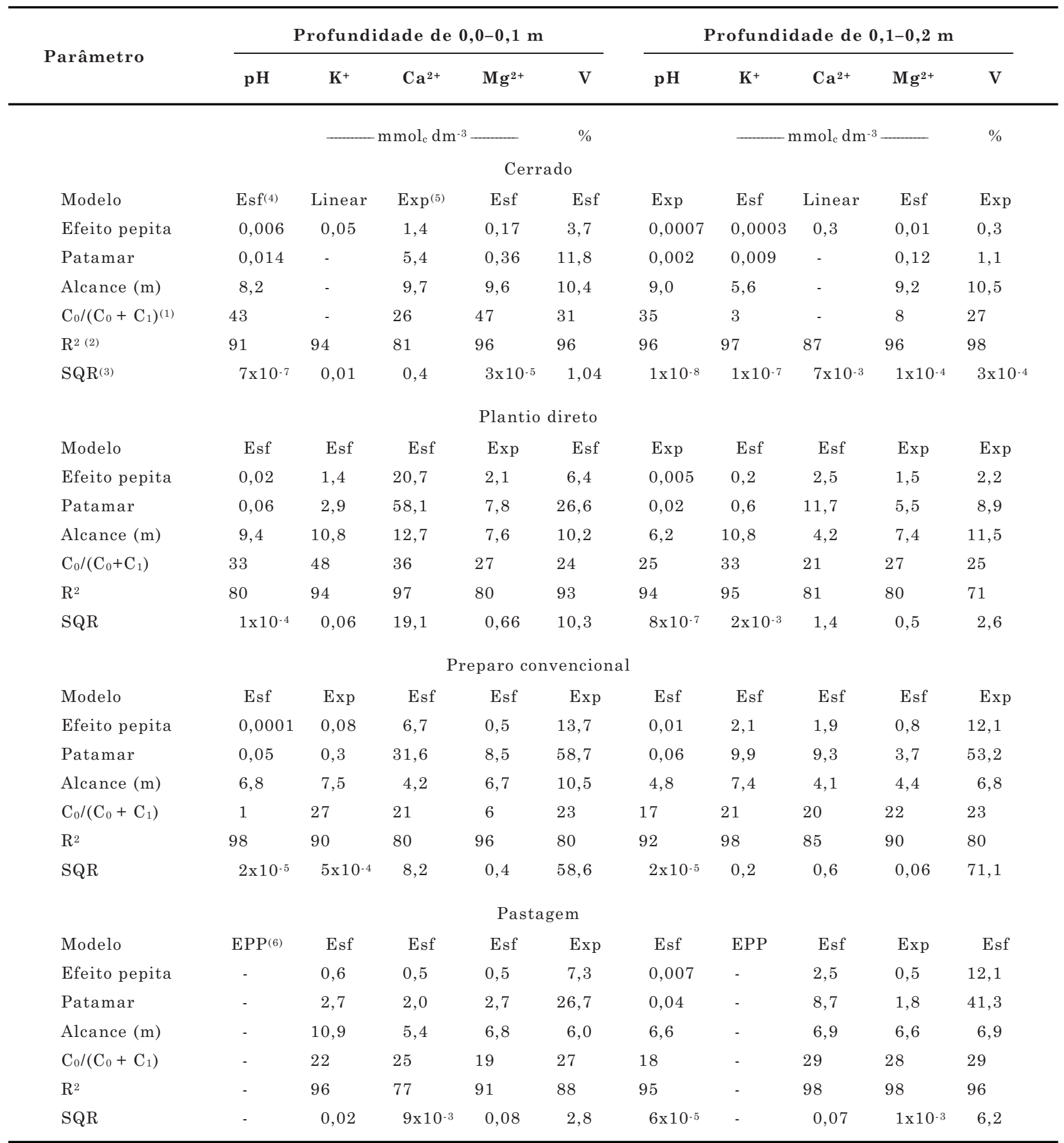

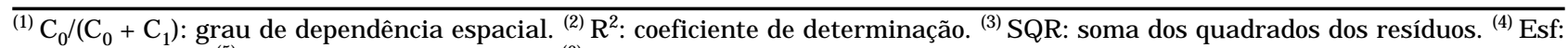
modelo esférico. ${ }^{(5)}$ Exp: modelo exponencial. ${ }^{(6)} \mathrm{EPP}$ : efeito pepita puro.

nas profundidades de $0,0-0,1$ e $0,1-0,2 \mathrm{~m}$, respectivamente, as quais apresentaram efeito pepita puro, indicando, segundo Cambardella et al. (1994), variabilidade não explicada, que pode ser devida a erros de medida e microvariações não detectadas, considerando a distância de amostragem utilizada
(Quadro 2 e Figuras 2, 3, 4 e 5). A análise dos semivariogramas para os atributos químicos em estudo não indicou nenhuma direção preferencial, ou seja, os dados não possuem anisotropia; a variabilidade espacial dos dados ocorre da mesma maneira em todas as direções (Vieira, 2000). 

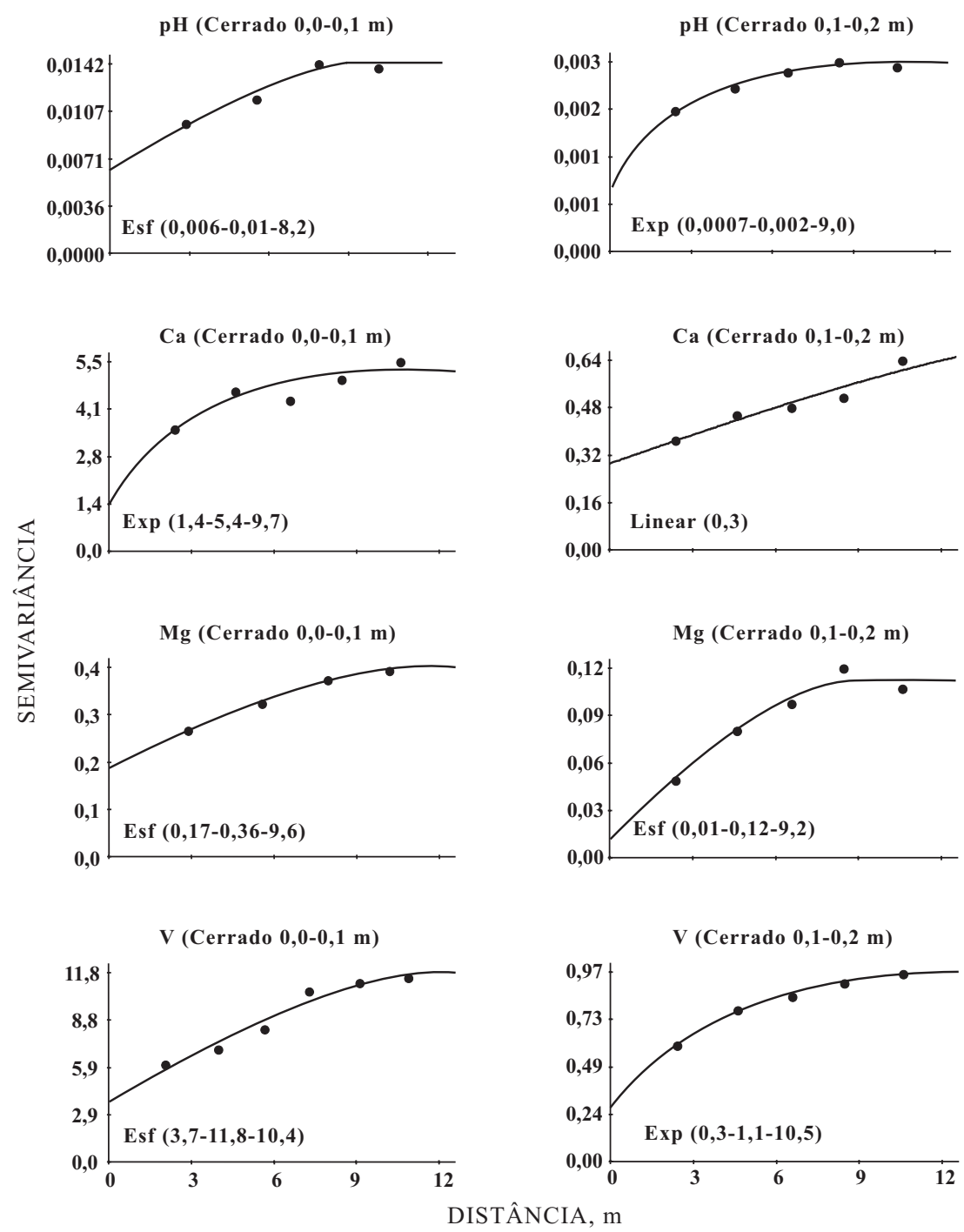

Figura 2. Semivariogramas dos atributos químicos do solo no Cerrado, nas profundidades de 0,0-0,1 e 0,1$0,2 \mathrm{~m}$.

Todos os semivariogramas ajustaram-se aos modelos esférico e exponencial, exceção para as variáveis $\mathrm{K}$ e Ca no Cerrado nas profundidades de 0,0-0,1 e 0,1-0,2 m, respectivamente, que se ajustaram ao modelo linear (Quadro 2). Esses modelos são considerados transitivos (Isaaks \& Srivastava, 1989), pois possuem patamar, ou seja, a partir de um determinado valor da distância entre amostras não existe mais dependência espacial (a variância da diferença entre pares de amostras torna-se invariante com a distância). Os modelos esféricos e exponenciais apresentam-se como os modelos teóricos mais comuns aos atributos do solo e da planta (Trangmar et al., 1985; Cambardella et al., 1994; Salviano et al., 1998; Carvalho et al., 2003).

Observa-se por meio do exame dos semivariogramas que o $\mathrm{pH}$ apresentou o menor efeito pepita, quando comparado com os demais atributos químicos em estudo, e também o menor CV (Quadros 1 e 2). Dessa maneira, é possível prever por meio da estatística descritiva e geoestatística os atributos do solo que apresentaram valores de variabilidade não detectada pelo esquema de amostragem, indicando maior descontinuidade entre amostras. Amostragens mais intensivas podem revelar maior continuidade espacial dos atributos químicos analisados.

O efeito pepita reflete a variabilidade não explicada em função da distância da amostragem utilizada, como variações locais, erros de análise, erros de amostragem e outros. Como é impossível quantificar a contribuição individual desses erros, o efeito pepita pode ser expresso como percentagem do patamar, facilitando, assim, a comparação do grau de dependência espacial das variáveis em estudo (Trangamar et al., 1985).

$\mathrm{Na}$ análise do grau de dependência espacial dos atributos químicos, utilizou-se a classificação de Cambardella et al. (1994). A relação $\mathrm{C}_{0} /\left(\mathrm{C}_{0}+\mathrm{C}_{1}\right)$ no 

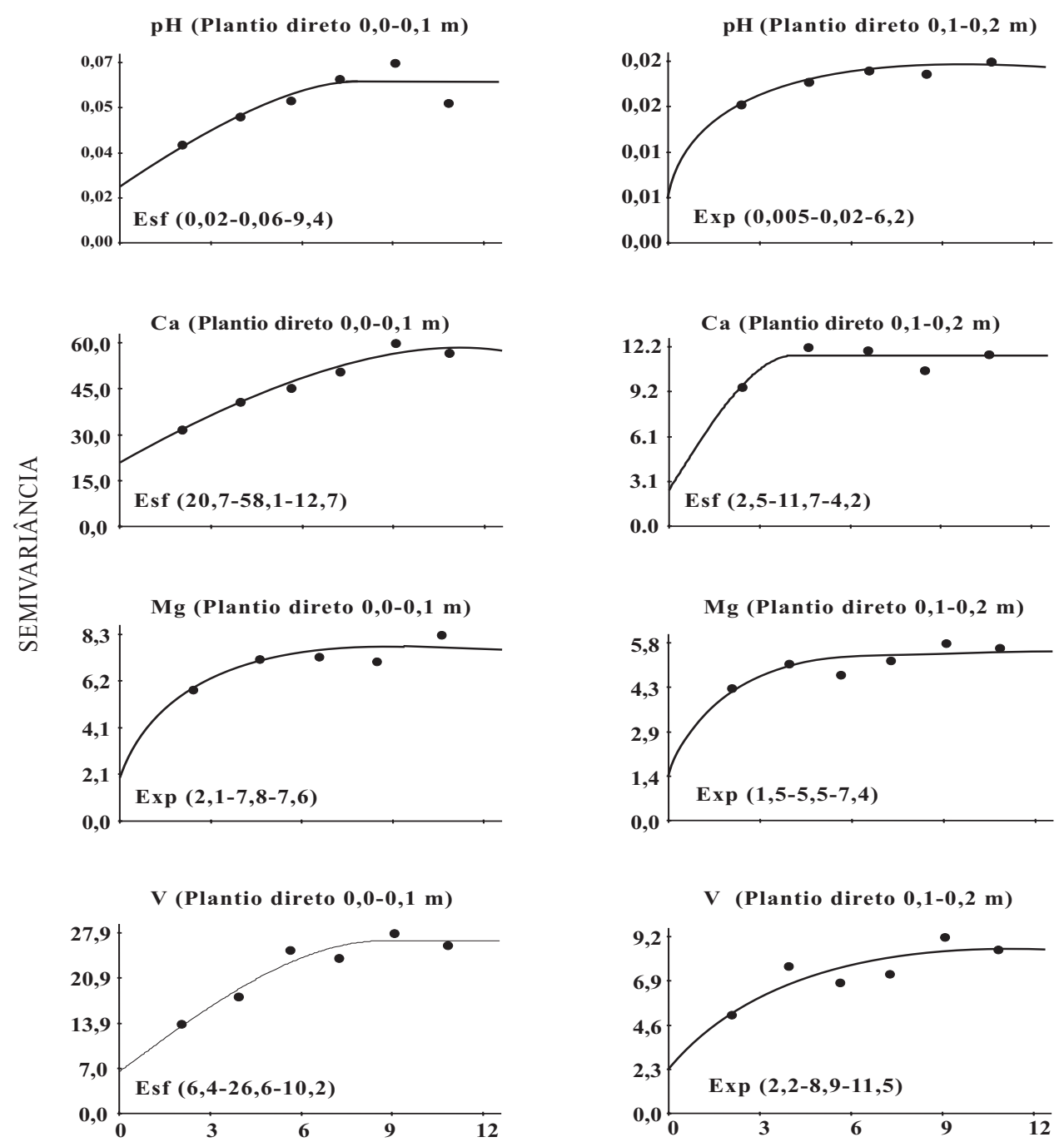

DISTÂNCIA, m

Figura 3. Semivariogramas dos atributos químicos do solo no sistema plantio direto, nas profundidades de 0,0-0,1 e 0,1-0,2 m.

Cerrado mostrou que todos os atributos químicos apresentaram dependência espacial com grau moderado, nas profundidades estudadas, com exceção do $\mathrm{K}$ e $\mathrm{Mg}$, que mostraram grau da dependência espacial forte na profundidade de 0,1-0,2 m (Quadro 2 e Figuras 2, 3, 4 e 5). No sistema plantio direto, o V, em ambas as profundidades estudadas, o $\mathrm{pH}$ e o $\mathrm{Ca}$ na profundidade de $0,1-0,2 \mathrm{~m}$ apresentaram dependência espacial forte. Verificou-se dependência espacial forte para todos os atributos químicos em estudo no sistema preparo convencional, em ambas as profundidades estudadas, com exceção do $\mathrm{K}$ na profundidade de 0,0-0,1 m, que mostrou dependência espacial moderada. Já na área com pastagem verificouse dependência espacial moderada para o $\mathrm{V}$, nas profundidades de 0,0-0,1 e 0,1-0,2 m, e o Ca e Mg na profundidade de $0,1-0,2 \mathrm{~m}$, bem como dependência espacial forte para $\mathrm{K}$, Ca e $\mathrm{Mg}$ na profundidade de $0,0-0,1 \mathrm{~m}$ e $\mathrm{pH}$ na profundidade de $0,1-0,2 \mathrm{~m}$.
Usualmente, a forte dependência espacial dos atributos do solo é atribuída aos fatores intrínsecos, ao passo que aos extrínsecos atribui-se fraca dependência (Cambardella et al., 1994). Portanto, a forte dependência espacial detectada para os atributos químicos em estudo pode ser decorrente de qualquer um dos fatores de formação do solo, principalmente o material de origem e relevo, enquanto que a dependência espacial moderada, seria devido a homogeneização do solo, que as adubações e calagens proporcionaram ao solo, nos diferentes sistemas de uso e manejo.

Os atributos químicos apresentaram diferentes alcances, nas profundidades de 0,0-0,1 e 0,1-0,2 m (Quadro 2 e Figuras 2, 3, 4 e 5). O Ca apresentou o maior alcance na profundidade de $0,0-0,1 \mathrm{~m}$ no sistema plantio direto $(12,7 \mathrm{~m})$ e o menor no sistema preparo convencional na profundidade de $0,1-0,2 \mathrm{~m}$ $(4,2 \mathrm{~m})$. Observou-se que os sistemas preparo 

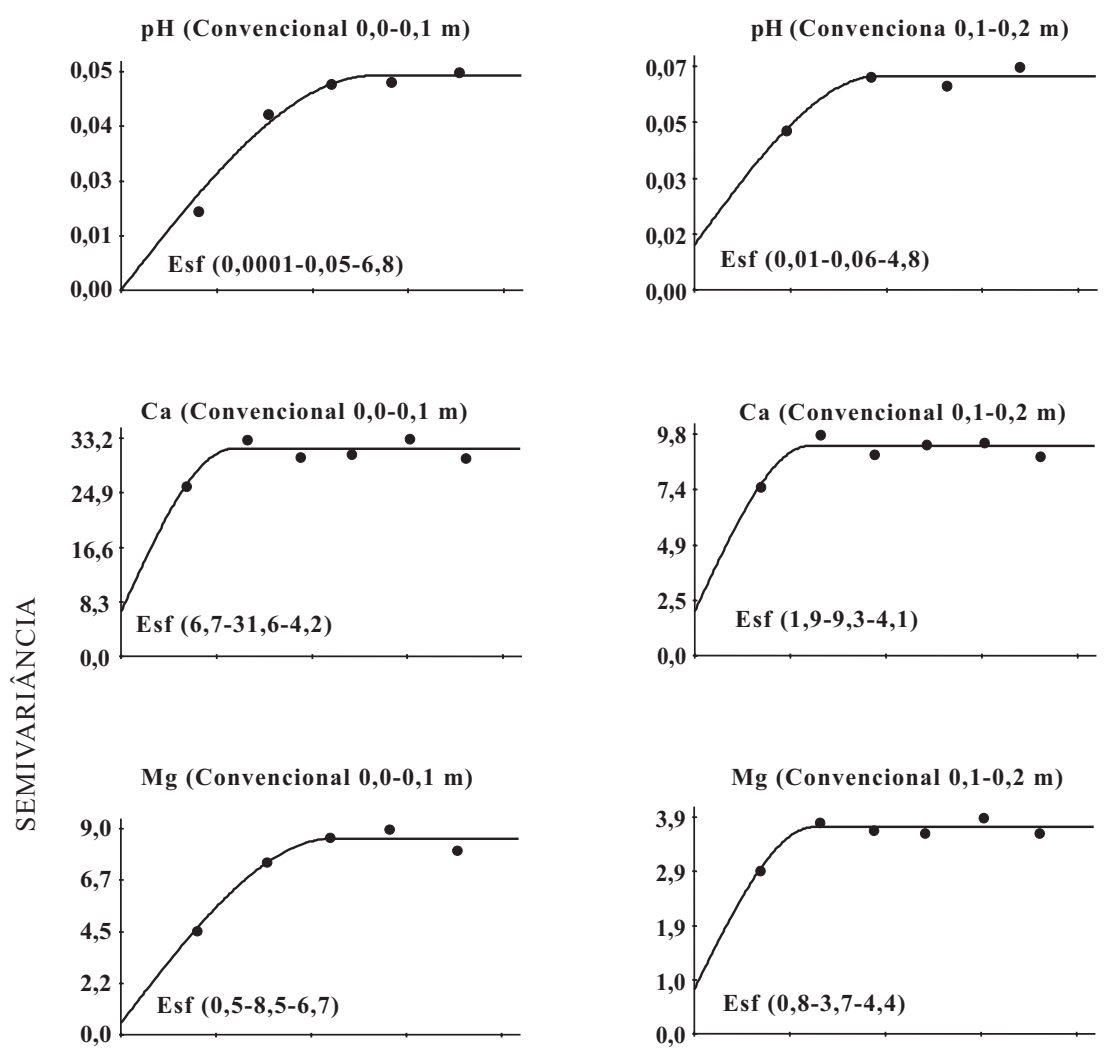

V (Convencional 0,0-0,1 m)

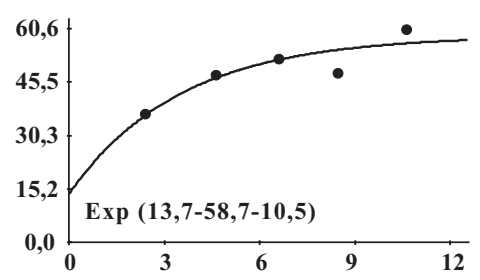

V (Convencional 0,1-0,2 m)

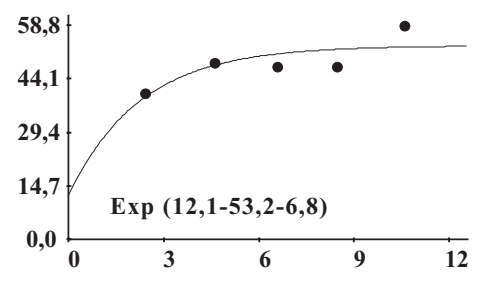

DISTÂNCIA, m

Figura 4. Semivariogramas dos atributos químicos do solo no sistema preparo convencional, nas profundidades de 0,0-0,1 e 0,1-0,2 m.

convencional e pastagem apresentaram os menores alcances, quando comparado a Cerrado e plantio direto, com exceção de $\mathrm{K}$ no preparo convencional e $\mathrm{V}$ na área com pastagem na profundidade de $0,0-0,1 \mathrm{~m}$. Resultado contrário foi observado por Souza et al. (1998), que, estudando a variabilidade espacial de atributos químicos em relação a sistemas de manejo, observaram que o sistema plantio direto teve os menores alcances, e o sistema pastagem, os maiores.

O alcance da autocorrelação espacial, ou seja, a distância máxima na qual os atributos estão espacialmente correlacionados, foi comparado para os diferentes usos do solo na área estudada. A dimensão desse parâmetro indica um raio dentro do qual os valores se apresentam com grande semelhança e são correlacionados. Observa-se que no sistema Cerrado os valores do alcance estão relativamente próximos, variando de 5,6 a 10, $5 \mathrm{~m}$ (Figura 2). No sistema plantio direto notou-se maior intervalo do alcance entre os atributos químicos estudados: 4,2 a 12,7 m (Figura 3), concordando com os resultados obtidos por Guimarães (2000), estudando a variabilidade de um Latossolo Vermelho submetido a plantio direto e convencional. Na área com plantio convencional observou-se certa semelhança entre os valores de alcance: 4,1 a 7,4 m (Figura 4), com exceção da variável $\mathrm{V}$ na profundidade de $0,0-0,1 \mathrm{~m}$, que mostrou maior alcance. O mesmo comportamento foi verificado na área com pastagem, com valores de alcance muito próximos, 6,0 a 7,0 m (Figura 5), com exceção do atributo $\mathrm{K}$ na profundidade de 0,0-0,1 m. Souza et al. (1997) sugerem, para futuras amostragens, que o intervalo entre amostras tenha valores menores que o alcance da dependência espacial. 

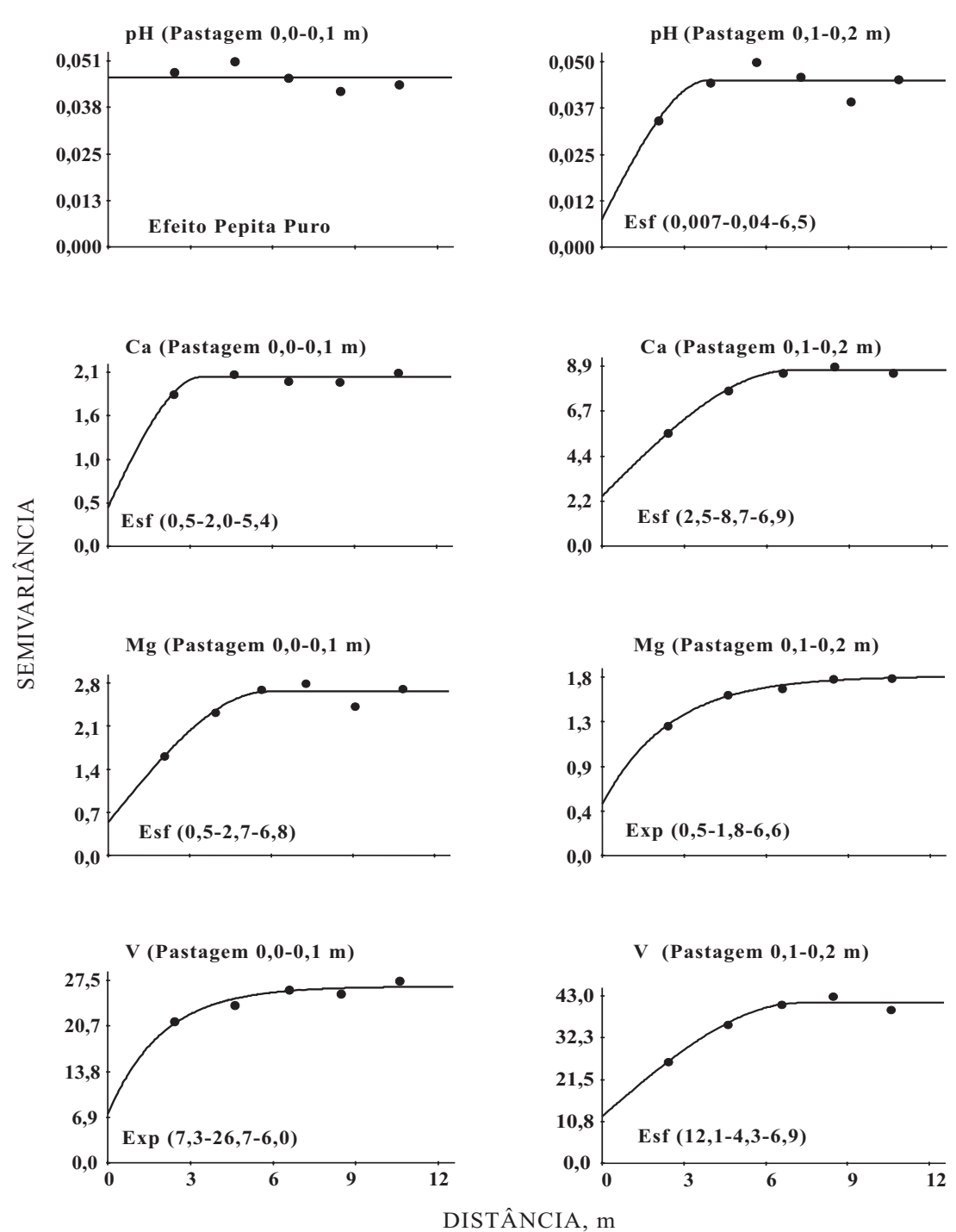

Figura 5. Semivariogramas dos atributos químicos do solo na área com pastagem, nas profundidades de 0,00,1 e 0,1-0,2 m.

\section{CONCLUSÃo}

O uso e manejo do solo realizado ao longo do tempo evidenciaram a variabilidade espacial dos seus atributos químicos nas profundidades estudadas.

\section{LITERATURA CITADA}

ALBUQUERQUE, J.A.; REINERT, D.J. \& FIORIN, J.E. Variabilidade de solo e planta em podzólico vermelhoamarelo. R. Bras. Ci. Solo, 20:151-157, 1996.

BAYER, C. \& MIELNICZUK, J. Nitrogênio total de um solo submetido a diferentes métodos de preparo e sistemas de cultura. R. Bras. Ci. Solo, 21:235-239, 1997.

BECKETT, P.H.T. \& WEBSTER, R. Soil variability: A review. Soil Fert., 34:1-15, 1971.
BHATTI, A.U.; MULLA, D.J. \& FRAZIER, B.E. Estimation of soil properties and wheat yields on complex eroded hills using geostatistics and thematic mapper images. Remote Sens. Environ., 37:181-191, 1991.

BOUMA, J.; STOORVOGEL, J.; ALPHEN, B.J. \& BOOLTINK, H.W.G. Pedology, precision agriculture, and the changing paradigm of agricultural research. Soil Sci. Soc. Am. J., 63:1763-1768, 1999 .

CAMBARDELLA, C.A.; MOORMAN, T.B.; NOVAK, J.M.; PARKIN, T.B.; KARLEN, D.L.; TURCO, R.F. \& KONOPKA, A.E. Field-scale variability of soil properties in Central Iowa Soils. Soil Sci. Soc. Am. J., 58:1501-1511, 1994.

CARVALHO, J.R.P.; SILVEIRA, P.M. \& VIEIRA, S.R. Geoestatística na determinação da variabilidade espacial de características químicas do solo sob diferentes preparos. Pesq. Agropec. Bras, 37:1151-1159, 2002.

CARVALHO, M.P.; TAKEDA, E.Y. \& FREDDI, O.S. Variabilidade espacial de atributos de um solo sob videira em Vitória Brasil (SP). R. Bras. Ci. Solo, 27:695-703, 2003. 
EMPRESA BRASILEIRA DE PESQUISA AGROPECUÁRIA EMBRAPA. Centro Nacional de Pesquisa de Solos. Sistema brasileiro de classificação de solos. Brasília, 1999. $412 \mathrm{p}$.

GUIMARÃES, E.C. Variabilidade espacial de atributos de um Latossolo Vermelho-Escuro textura argilosa da região de cerrado, submetido ao plantio direto e ao plantio convencional. Campinas, Universidade Estadual de Campinas, 2000. 89p. (Tese de Doutorado)

ISAAKS, E.H. \& SRIVASTAVA, R.M. An introduction to applied geoestatistics. New York, Oxford University Press, 1989. $561 \mathrm{p}$.

JACOB, W.L. \& KLUTTE, A. Sampling soils for physical and chemical properties. Soil Sci. Soc. Am. Proc., 20:170-178, 1976.

LITTLE, T.M. \& HILLS, F.J. Agricultural experimentation. New York, John Wiley \& Sons, 1978. 350p.

RAIJ, B. van; ANDRADE, J.C.; CANTARELLA, H. \& QUAGGIO, J.A., eds. Análise química para avaliação da fertilidade de solos tropicais. Campinas, Instituto Agronômico, 2001. 285p.

RAIJ, B. van; CANTARELLA, H.; QUAGGIO, J. A. \& FURLANI, A.M.C. Recomendações de adubação e calagem para o Estado de São Paulo. 2.ed. Campinas, Instituto Agronômico/Fundação IAC, 1997. 285p. (Boletim Técnico, 100)

ROBERTSON, G.P. GS+: Geostatistics for the environmental sciences - $\mathrm{GS}^{+}$User's guide. Plainwell, Gamma Design Soffware, 1998. 152p.

SALVIANO, A.A.C.; VIEIRA, S.R. \& SPAROVEK, G. Variabilidade espacial de atributos de solo e de Crotalaria juncea $(L)$ em área severamente erodida. R. Bras. Ci. Solo, 22:115-122, 1998.
SCHLOTZHAVER, S.D. \& LITTELL, R.C. SAS: System for elementary statistical analysis. 2.ed. Cary, SAS, 1997.

SILVA, V.R.; REICHERT, J.M.; STORCK, L. \& FEIJÓ, S. Variabilidade espacial das características químicas do solo e produtividade de milho em um Argissolo VermelhoAmarelo distrófico arênico. R. Bras. Ci. Solo, 27:10131020, 2003.

SOUZA, L.S.; COGO, N.P. \& VIEIRA, S.R. Variabilidade de fósforo, potássio e matéria orgânica no solo, em relação a sistemas de manejo. R. Bras. Ci. Solo, 22:77-86, 1998.

SOUZA, L.S.; COGO, N.P. \& VIEIRA, S.R. Variabilidade de propriedades físicas e químicas do solo em um pomar cítrico. R. Bras. Ci. Solo, 21:367-372, 1997.

SOUZA, Z.M. \& ALVES, M.C. Propriedades químicas de um Latossolo Vermelho distrófico de cerrado sob diferentes usos e manejos. R. Bras. Ci. Solo, 27:133-139, 2003.

TRANGMAR, B.B.; YOST, R.S.; WADE, M.K. \& UEHARA, G. Applications of geostatistics to spatial studies of soil properties. Adv. Agron., 38:45-94, 1985.

VANNI, S.M. Modelos de regressão: Estatística aplicada. São Paulo, Legmar Informática, 1998. 177p.

VIEIRA, S.R. Geoestatística em estudos de variabilidade espacial do solo. In: NOVAIS, R.F.; ALVAREZ V., V.H. \& SCHAEFER, C.E.G.R., eds. Tópicos em ciência do solo. Viçosa, MG, Sociedade Brasileira de Ciência do Solo, 2000. v.1. p.1-53.

VIEIRA, S.R.; HATFIELD, J.L.; NIELSEN, D.R. \& BIGGAR, J.W. Geoestatiscal theory and application to variability of some agronomical properties. Hilgardia, 51:1-75, 1983.

WARRICK, A.W. \& NIELSEN, D.R. Spatial variability of soil physical properties in the field. In: HILLEL, D., ed. Applications of soil physics. New York, Academic Press, 1980. p.319-344. 